\title{
The exploration-exploitation trade-off in a foraging task is affected by mood-related arousal and valence
}

\author{
Roel van Dooren ${ }^{1} \cdot$ Roy de Kleijn ${ }^{1} \cdot$ Bernhard Hommel $^{1} \cdot$ Zsuzsika Sjoerds $^{1}$
}

Accepted: 11 May 2021 / Published online: 4 June 2021

(C) The Author(s) 2021

\begin{abstract}
The exploration-exploitation trade-off shows conceptual, functional, and neural analogies with the persistence-flexibility tradeoff. We investigated whether mood, which is known to modulate the persistence-flexibility balance, would similarly affect the exploration-exploitation trade-off in a foraging task. More specifically, we tested whether interindividual differences in foraging behavior can be predicted by mood-related arousal and valence. In 119 participants, we assessed mood-related interindividual differences in exploration-exploitation using a foraging task that included minimal task constraints to reduce paradigm-induced biases of individual control tendencies. We adopted the marginal value theorem as a model-based analysis approach, which approximates optimal foraging behavior by tackling the patch-leaving problem. To assess influences of mood on foraging, participants underwent either a positive or negative mood induction. Throughout the experiment, we assessed arousal and valence levels as predictors for explorative/exploitative behavior. Our mood manipulation affected participants' arousal and valence ratings as expected. Moreover, mood-related arousal was found to predict exploration while valence predicted exploitation, which only partly matched our expectations and thereby the proposed conceptual overlap with flexibility and persistence, respectively. The current study provides a first insight into how processes related to arousal and valence differentially modulate foraging behavior. Our results imply that the relationship between exploration-exploitation and flexibility-persistence is more complicated than the semantic overlap between these terms might suggest, thereby calling for further research on the functional, neural, and neurochemical underpinnings of both trade-offs.
\end{abstract}

Keywords Exploration $\cdot$ Exploitation $\cdot$ Flexibility $\cdot$ Persistence $\cdot$ Mood $\cdot$ Foraging

\section{Introduction}

The adaptivity of human behavior often is attributed to cognitive control, the ability to tailor basic cognitive abilities to the task and situation at hand. Adaptive control often requires the solution of control dilemmas (Goschke, 2003), of which several have received substantial attention in the literature. Among those are the exploration-exploitation dilemma-

Online Supplementary Material can be found at: https://osf.io/bm6ps/. We made our data, material, and code (licensed under a Creative Commons Attribution-NonCommercial 4.0 International License) available at: https://osf.io/skh5y/

Roel van Dooren roelvdooren@gmail.com

1 Cognitive Psychology Unit, Institute of Psychology \& Leiden Institute for Brain and Cognition, Leiden University, Wassenaarseweg 52, 2333 AK Leiden, The Netherlands addressing the question whether one should continue using the currently available knowledge or seek for new information - and the stability/persistence-flexibility dilemma (or persistence-flexibility dilemma for short) — tackling the question whether one should stick to one's current goal or trade it for another, more tempting or easier to achieve alternative. In the present study, we were interested in possible functional commonalities between these control trade-offs.

We were motivated by the observation that the explorationexploitation and persistence-flexibility dilemmas share many characteristics with respect to their descriptions in the literature. For one, the theoretical concepts of exploration and flexibility both imply that the present control settings need to be overruled and that the competitive strength of alternatives is increased, whereas the concepts of exploitation and persistence both refer to the continued maintenance of the current control settings in the more or less active suppression of alternative settings. Indeed, previous work within the domain of cognitive psychology has not drawn a sharp line between 
exploration-exploitation on the one hand and flexibilitypersistence on the other (Hills, 2006; Hills \& Dukas, 2012; Hommel \& Colzato, 2017; Marković et al., 2019). For instance, and among others, Hills et al. $(2008,2010)$ have illustrated that such theoretical overlap is not far-fetched, because abstract conceptual search can be primed by inducing explorative or exploitative foraging strategies, with high levels of exploration (vs. exploitation) boosting flexible (vs. persistent) cognitive search patterns.

Along similar lines, even though both trade-offs have been addressed from different computational accounts, functional overlap between these control dilemmas can be inferred. For instance, the exploration-exploitation dilemma is often discussed in the context of (food) foraging (Wolfe, 2013). Exploiting an area that provides known and preferred food or rewards is typically energy-saving, as prediction-error signals decrease with increased expectation-outcome matches. However, at a particular point in time, provided that the availability of food or reward is subject to time and/or consumption, it might become more efficient to invest time and effort into moving to another area and start exploring for new resources (however, see Riefer et al., 2017). Hence, to remain efficient and keep the energy cost optimally low, foraging calls for an adaptive strategy in balancing the explorationexploitation trade-off. In the same vein, an adaptive strategy can be distilled from the conceptualization of the persistenceflexibility trade-off. Specifically, having selected and persistently implemented a goal, task, or action is not sufficient for guaranteeing smooth performance, and might be increasingly difficult or mentally costly to maintain, especially in the presence of external stimuli or internal thoughts that promote other, competing goals, tasks, or actions (Atkinson \& Birch, 1970). Protecting the current action goal against such interference requires active goal shielding, which would need to result in the suppression of alternative goals and respective response tendencies (Dreisbach \& Goschke, 2004). However, in changing environments it may not always be the most optimal strategy to persist in the current action goal. Under such conditions, it would be adaptive to open up for other options that might outcompete the present goal- to become more flexible that is, reflected in a weak influence of the current goal on selection and weak competition between alternative codes (Hommel, 2015; Hommel \& Colzato, 2017). Therefore, the exploration-exploitation and persistence-flexibility dilemma's might be comparable in the fact that adaptive control is required to find an appropriate balance between choosing between familiar, but sometimes suboptimal alternatives and novel, often risky alternatives (Cohen et al., 2007).

Finally, in addition to these conceptual, and perhaps even functional overlaps, commonalities regarding the assumed neural mechanisms underlying the solution of the two dilemmas could similarly be distilled (Hills, 2006). For instance, tonic levels of norepinephrine (NE), reflecting more enduring and less discriminative neural responsiveness (Aston-Jones \& Cohen, 2005), have been claimed to reduce the explorationexploitation dilemma by promoting exploratory behavior (Jepma \& Nieuwenhuis, 2011). Analogously, tonic NE levels have been suggested to play a role for the persistenceflexibility trade-off by biasing perception and memory in favor of salient, high priority representations (possibly reflecting increased persistence) at the expense of lower priority representations (possibly reflecting reduced flexibility; Mather et al., 2016). Similarly, dopamine (DA) has been suggested to play a central role in resolving both the explorationexploitation trade-off (Cohen et al., 2007; Frank et al., 2009; Frank \& Fossella, 2011; Hills, 2006; Hills et al., 2010; Kayser et al., 2015) as well as the persistence-flexibility dilemma (Cools \& d'Esposito, 2011; Dreisbach et al., 2005; Dreisbach \& Goschke, 2004; Durstewitz \& Seamans, 2008; Hommel \& Colzato, 2017).

Altogether, these commonalities seem to suggest that the semantic, functional, and perhaps even neural mechanisms responsible for finding the best balance between exploration and exploitation might, at least to some extent, overlap with those responsible for balancing flexibility and persistence. We admit that by theoretically equating these concepts, we do not do full justice to the tremendous amount of work that has been dedicated to the systematic study of both control dilemmas. For instance, research within the domain of decision making has shown that the operationalization of exploration and exploitation is dependent on the field of study, especially when it comes to its application in foraging behavior. While models of the explore-exploit dilemma differentiate between explicitly seeking out information versus acting on known rewards (Cohen et al., 2007), models of foraging center on probabilistically estimating average- and current reward rates (Charnov, 1976). However, even though these conceptualizations are often conflated (Todd \& Hills, 2020), this does not render the core premise any less valid. In both instances, the goal of the organism is to maximize reward. In any case, we point out that a full-fledged discussion on these exact operationalizations, as well as a formalization of consensual definitions, is beyond the scope of this study and admit that our understanding of the two trade-offs as sharing semantic, functional, and neural commonalities is not necessarily consistent with all available explicit and implicit definitions of exploration/exploitation and persistence/flexibility. Taking these caveats into consideration, we speculated that factors that are known to have a systematic impact on people's tendency to engage in more flexible or more persistent behavior should have a similar effect on the probability to show more explorative or more exploitative behavior.

Here we focused on a factor that has successfully been used to bias behavior away from persistence towards greater flexibility: mood. While the personal experience of mood is unlikely to have a causal impact on cognitive control processes 
(Hommel, 2019), the neurochemistry underlying mood changes has been found to systematically covary with changes in control policies. Moreover, induced positive mood, compared with negative or neutral mood, has been shown to promote cognitive flexibility and reduce persistence in a cognitive set-switching task (Dreisbach \& Goschke, 2004), to reduce goal refreshing after conflict trials (Van Steenbergen et al., 2010), to promote divergent thinking in a creativity task (Akbari Chermahini \& Hommel, 2012), and to reduce proactive control in a working memory updating task (Fröber \& Dreisbach, 2014). Provided that all these observations can be validly taken to indicate that positive mood tends to shift control away from persistence and towards greater flexibility (Dreisbach \& Goschke, 2004; Goschke \& Bolte, 2014; Hommel \& Colzato, 2017), we hypothesized that-if exploration-exploitation would show overlap conceptually and mechanistically with flexibility-persistence - inducing positive mood before presenting a task that is sensitive to exploration-exploitation tendencies should generate a stronger bias towards exploration. Such expectations are in line with observations as reported within the domain of affect and decision making, in which positive-going mood states have been shown to favor exploratory behavior (Vinckier et al., 2018), while negative-going mood states potentially result in overexploitation (Lenow et al., 2017).

Provided that mood induction is known to affect both perceived arousal and perceived valence measures, we also were interested to see whether these measures would differentially affect possible biases in exploration/exploitation, especially because arousal and valence have received a considerably different emphasis within accounts on the explorationexploitation trade-off compared with literature on the persistence-flexibility balance. Specifically, according to Cohen et al. (2007), NE, which is assumed to be associated with mood-related arousal (Terbeck et al., 2016), plays an important role in regulating exploration and exploitation. In contrast, in persistence-flexibility accounts, valence is often explicitly or implicitly assumed to be the key player (Van Steenbergen et al., 2010).

Taken altogether, the main goal of the present study was to test whether and how the mood-related regulation of the exploration-exploitation balance relates to the regulation of the persistence-flexibility trade-off. To this end, we designed a foraging task that was adopted from Hills et al. $(2008,2010)$, who originally had used it to induce explorative and exploitative foraging strategies. Given that we did not intend to induce or promote a particular control mode, but rather to assess the way our mood induction manipulation would shift the relative emphasis on exploration and exploitation, we adjusted the task to provide participants the freedom to explore or exploit the task environment following their individual tendencies instead of specific task-conditions. We compared performance in this foraging task pre-mood induction with performance after positive versus negative mood induction, which we assumed to modulate the trade-off between exploration and exploitation the same way as it has been shown to modulate the balance between persistence and flexibility.

In particular, we tested the following hypotheses: First, and foremost, we expected mood induction to affect the exploration-exploitation trade-off. Second, given that positive mood has been found to promote flexible task performance (Dreisbach \& Goschke, 2004; Fröber \& Dreisbach, 2014; Van Steenbergen et al., 2010), we expected a positive-going mood to promote exploration, whereas a negative-going mood should promote exploitation. Finally, given the differential roles of arousal and valence in modulating the explorationexploitation versus persistence-flexibility trade-offs, we tested whether arousal, valence or both measures would orthogonally modulate foraging behavior.

\section{Methods}

\section{Participants}

A total of 119 participants ( 78 females; $M_{\text {age }}=21.83, S D_{\text {age }}=$ 1.97) was recruited via the Research Participation System of the Leiden Institute of Psychology (Leiden University, Netherlands). Participants were in good mental and physical health and did not have a history of psychiatric or neurological conditions (as assessed by the Mini International Neuropsychiatric Interview; Sheehan et al., 1998), had normal or corrected-to-normal eyesight and were between ages 18 and 27 years. Participants provided written, informed consent and were naïve to the purpose of the experiment. An experimental session took approximately 45 minutes. In return for their participation, participants received either 2 course credits or $€ 5$. The study protocol was approved by the local ethics committee (Leiden University, Institute of Psychological Research; CEP19-0221/116).

Due to the novelty of our approach, it was challenging to properly estimate the best sample size before data collection. We did consult literature incorporating similar mood induction procedures in combination with cognitive control assessment (Jefferies et al., 2008; Van Steenbergen et al., 2010), which informed us that such manipulations typically include 20-25 participants per condition. However, such small samples sizes likely result in low statistical power. Indeed, we calculated that with an effect size of $\eta_{\mathrm{p}}{ }^{2}=0.05$ (Van Steenbergen et al., 2010, personal correspondence) a power of $<0.5$ would be obtained. Therefore, based on the effect sizes estimated from Van Steenbergen et al. (2010), we performed $a$ priori power calculations in GPower (Erdfelder et al., 1996) to estimate the minimum sample size for this study. Results indicated that a total sample size of 115-120 participants would be sufficient to detect such low effect sizes (i.e., $\eta_{\mathrm{p}}{ }^{2}=0.05$ ) 
with a power of 0.80 , and an alpha level of 0.05 (one-sided). Additionally, we included Bayesian analysis methods, because such approaches allow for explicit testing of the null versus the alternative hypothesis and provide possibilities for the post-hoc assessment of statistical power. Notably, we omitted data of two participants, because these participants indicated that they did not understand the structure of the foraging task, and hence showed inactivity in the task for a substantial amount of time. Consequently, the sample size for our analyses was reduced to a total of 117 participants.

\section{Procedure}

After arrival, participants were comfortably seated in front of a computer, at a distance of approximately $60 \mathrm{~cm}$ from the screen (AOC I2475PXQU monitor: $1920 \times 1080,23.8$ inch). They read and signed the informed consent, and demographics were assessed through standardized questions. Onscreen instructions guided them through a sequence of four tasks: (1) a practice navigation task, (2) a pre-mood induction run of the foraging task, (3) a mood induction procedure (excited vs. sad, counterbalanced between participants), and (4) a post-induction run of the foraging task. In order to dynamically capture changes in mood state, a $9 \times 9$ arousal by valence mood grid (Russell et al., 1989) was presented at multiple time points throughout the experiment. Upon completion of the experiment participants were debriefed and received their payment.

\section{Materials}

Practice navigation task Before the presentation of the experimental sequence, participants completed a navigation task to familiarize themselves with the joystick they would use within the foraging task runs. To this end, participants had to move a character $(19 \times 23$ pixels $)$ through a two-dimensional maze $(600 \times 600$ pixels $)$. The character's speed (0-60 pixels per second) and angle $\left(0^{\circ}-359^{\circ}\right)$ could be adjusted by means of the joystick. The goal was to successfully navigate the character to the exit of the maze. The trial was reinitialized if participants hit any of the maze's walls. No time restrictions were set. Average duration spent on this practice task was 23.05 seconds $(S D=8.07$ seconds $)$.

Foraging task The design of our foraging paradigm was inspired by the foraging task as described by Hills et al. (2008, 2010). However, while the original task by Hills and colleagues included two separate conditions aimed at inducing either exploitative or explorative foraging behaviour betweensubjects (i.e., clumpy vs. diffuse resource distributions, respectively), the set-up of our task was aimed at measuring individual foraging tendencies within-subjects. Therefore, we designed a task with as few task constraints as possible to reduce paradigm-related impact, enabling us to measure individual's, task-unconstrained tendency to explore versus exploit.

To effectively capture dynamic adjustments in foraging strategies, we provided participants with a "zoom-in to patch view/zoom-out to aerial view" feature, which allowed them to approach the foraging task as preferred. Specifically, this feature enabled participants to, at their own pace and as much as they preferred, freely explore the foraging environment in order to locate new resource patches, or return to already discovered resource patches. Moreover, it allowed participants to start exploiting previously unentered resource patches, or to start re-exploiting previously entered resource patches. Hence, we assume that the small adjustments we made with respect to the original task enable us to measure inter-individual differences in foraging strategies, with foraging behavior largely depending on individuals' internal foraging preference or strategy, which one could continuously update (e.g., from exploration to exploitation), unconstrained by task conditions. The foraging paradigm was coded in Python 2.7.

Participants were instructed to find as many resources (i.e., berries) within a restricted period of 5 minutes. Resources were hidden in patches (i.e., bushes), which in turn were hidden in a game environment $(600 \times 600$ pixels $)$. Each trial comprised nine unique patches (radii of 57 pixels), each constituting 40 to-be-discovered resources. The patch-positions were randomly drawn from two uniform distributions (for the $\mathrm{x}$ - and $\mathrm{y}$-coordinates, respectively), with the notable exceptions that (i) the minimum distance between patches was set to 9 pixels, (ii) patches were to be located at a minimum distance of 15 pixels from any of the environment's borders, and (iii) the minimum distance between the center of the environment and a patch constituted at least 57 pixels. The distribution of the patches thereby conceptually mirrored the clumpy resource distribution as described by Hills et al. (2008, 2010).

At the start of a trial, participants were presented with a game environment depicting this so-called aerial view (Fig. 1a). A character $(19 \times 23$ pixels $)$ was positioned in the center of the environment, facing a random location in space. The character could be moved by means of the joystick with a speed of 0-60 pixels per second and an angle of $0^{\circ}-359^{\circ}$. If the character was navigated to a position where a patch was hidden, the part of the patch that was present within a 15-pixel radius of the character's center location became visible. Once a patch was encountered, it could be entered by pressing a key on the back-side of the joystick. Upon patch entry, a new game environment $(600 \times 600$ pixels $)$ was rendered, which displayed a zoomed-in view of the entered patch (i.e., patch view; Fig. 1b). Within each patch, resources $(15 \times 15$ pixels $)$ were randomly distributed, with the precondition that resources were not allowed to be in a 30-pixel vicinity of any other resource. Hence, the distribution of resources within 


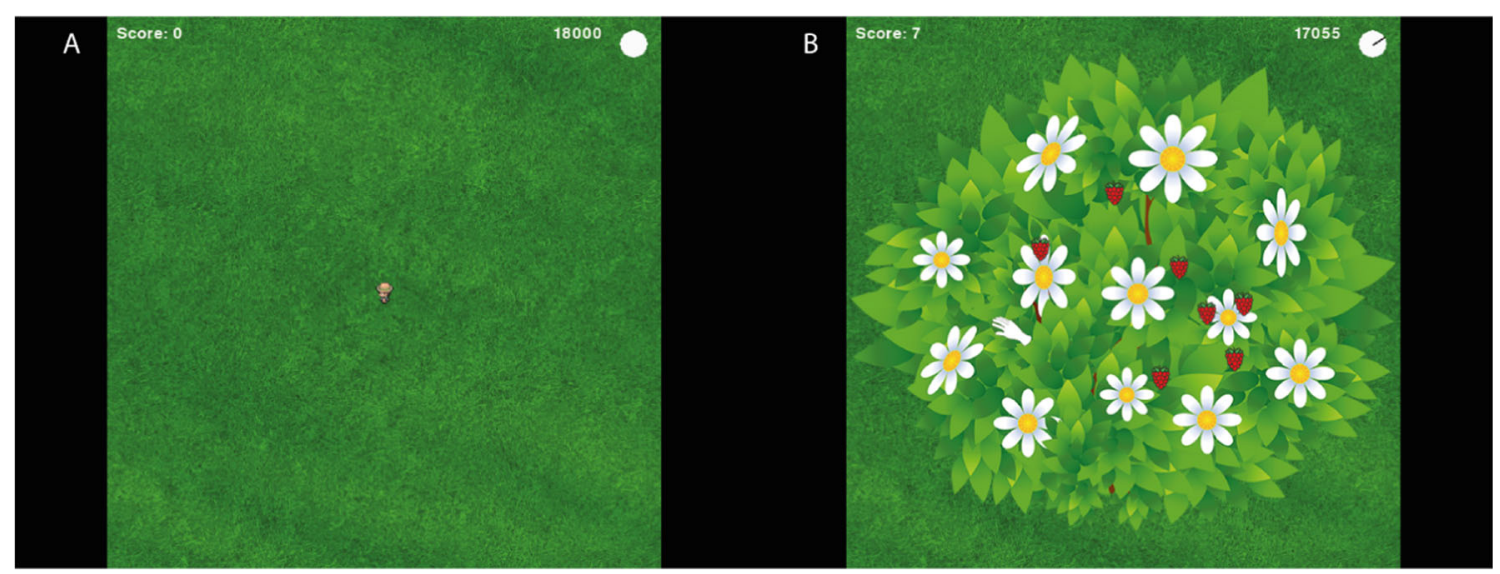

Fig. 1 Visual representation of the two layers of the foraging task, displaying the aerial-view (a) and patch-view (b)

patches conceptually mirrored the diffuse resource distribution as described by Hills et al. $(2008,2010)$. The character was replaced by the image of a hand $(49 \times 44$ pixels $)$, which could, by means of the joystick, be moved with a speed of 0 90 pixels per second and an angle of $0^{\circ}-359^{\circ}$. If the hand was navigated to a position where a resource was hidden (i.e., within a 30-pixel radius of the hand's center location), the resource became visible. Each found resource, equaling 1 point, was displayed on screen as a red berry $(33 \times 33$ pixels $)$. Importantly, before the start of the foraging trial, participants were informed that resources could only be found once a patch was entered (i.e., in patch view), while new patches could only be discovered in aerial view. Moreover, they were informed that the three participants that, overall, collected most resources, were rewarded with an additional voucher of $€ 15$.

In line with the task design of Hills et al. $(2008,2010)$, a counter was presented in the top-right corner of the environment. The counter started at a value of 18,000 (i.e., the maximum speed in pixels per second within the aerial view multiplied by the 300 -second duration of a foraging run) and decreased with a value of 60 units per second. Adjacent to this counter, a sweeping clock-hand was presented. Together, these tools informed participants that time was running out, without providing direct information on the actual time that remained. Finally, the total amount of resources found was continually updated and presented in the upperleft corner of the screen.

Marginal value theorem To investigate participants' general tendencies to explore/exploit in the foraging task, we adopted the marginal value theorem (MVT; Charnov, 1976), which aims to characterize and provide an approximation of optimal foraging behavior by tackling the patch-leaving problem.

The marginal value theorem posits that during foraging in a patchy environment, the amount of food gained for time $T$ spent in a patch is $f(T)$, with $f(T)$ rising from zero at a negatively accelerated rate to an asymptote representing the total amount of food present in a patch. As the first derivative of
$f(T)$, the current rate of food intake, approaches zero, an organism will not derive any benefit from staying in that particular patch as it will have exploited all its resources. When exactly should an organism move away from its current patch to the next? In the original case of multiple patch types, the average time $T$ spent to use one patch is the travel time plus the time spent in the patch:

$T=t+P_{A} \cdot T_{A}+P_{B} \cdot T_{B}$

and the average amount of food $E$ taken from a patch is

$E=P_{A} \cdot f\left(T_{A}\right)+P_{B} \cdot f\left(T_{B}\right)$

in which $P_{A}$ and $P_{B}$ are the proportion of patches visited that are of type A or B. In the current paradigm, this can be simplified to

$T=t+T_{P}, E=f\left(T_{P}\right)$

in which $T_{P}$ is the time spent in any patch. It follows then that $\mathrm{E} / \mathrm{T}$ reflects the average rate of food intake, which an organism should try to maximize. To find this maximum, we can find the partial derivative of $\mathrm{E} / \mathrm{T}$ using the quotient rule, setting

$$
\frac{\delta(E / T)}{\delta T_{P}}=0,
$$

we then find that this is the case when

$$
\frac{\delta f\left(T_{P}\right)}{\delta T_{P}}=E / T
$$

or, in other words, when the current food intake rate $f\left(T_{P}\right)$ is identical to the average food intake rate $\mathrm{E} / \mathrm{T}$. In the current paper, we will refer to these variables as the current reward rate CRR and the average reward rate ARR, respectively. Following MVT, exploitation should be preferred over exploration whenever the CRR is higher than the ARR. In other words, one should shift from an exploitative to a more exploratory foraging mode whenever the instantaneous reward rate 
becomes lower than the average expected reward rate. Because in the present paradigm rewards are received in a discrete instead of a continuous manner (i.e. the actual received reward is either 0 or 1 ), the CRR parameter was calculated over a short time interval.

Optimal window for calculating the CRR To determine an interval over which to calculate the CRR, we iteratively fitted the data of the first foraging run of each participant for multiple time windows (Supplementary Figure 1). Our fitting procedure extracted, separately for each participant and each patch visit, the interval for which, on average, the difference between the optimal leave time (OLT) and participants' actual leave time (ALT) was closest to 0 (i.e., leave time difference; LTD). In Fig. 2, OLTs are reflected as moments where the CRR and ARR intersect after a peak in the CRR signal is detected.

By adopting this normalization step, deviations from 0 on the LTD parameter on the foraging run following the mood induction procedure would thereby likely reflect effects induced by the manipulation, with a negative LTD reflecting an emphasis on exploitation over exploration (OLT < ALT) and a positive LTD implying a more exploratory foraging mode (OLT > ALT). We acknowledge that this is only one method for determining what interval to use to determine the $\mathrm{CRR}$, but considering we are interested in changes to foraging behavior the exact definition of CRR should not matter.

Within the first iteration, the LTD parameter was calculated for a total of 200 unique time windows $(0-20$ seconds in steps of 100 milliseconds). The analysis revealed that, on average, LTD was smallest for an interval of exactly 9.00 seconds (i.e., OLT - ALT $=-0.024)$. To confirm that we extracted the most optimal time window over which to calculate the CRR, we repeated this procedure in a second iteration by estimating the
LTD parameter for another 50 unique time windows (8.759.25 seconds in steps of 10 milliseconds). Once again, the analysis revealed that, on average, LTD was smallest for the 9.00-second time window. Importantly, a between-subjects ANOVA confirmed that, pre-mood induction, the LTD parameter for participants in the excited and sad mood induction conditions (see below) did not significantly differ $(F(1,115)=$ $\left.0.30, p=0.59, \mathrm{np}^{2}<0.01\right)$.

Mood induction The mood induction protocol used music to manipulate mood, a procedure that has successfully been used before (Eich et al., 2007; Van Steenbergen et al., 2010). Our induction protocol incorporated two conditions to which participants were randomly assigned: a positive (excited; high arousal, high valence) or negative (sad; low arousal, low valence) induction condition ( $\left.n_{\text {excited }}=57, n_{\mathrm{sad}}=60\right)$. During the mood induction procedure, participants listened to classical music congruent with the mood condition whilst writing down a memory or hypothetical situation in which they have experienced or would hypothetically experience the intended mood of the condition. The design was a double-blind study design, in the way that participants were not informed beforehand that we tested two different mood conditions, and the experimenter did not know the condition participants were in, as the induction happened using headphones, and the written stories were not read by the experimenter. The mood induction phase lasted 10 minutes. Participants in the sad condition that indicated to still be in a negative mood during the debriefing were given the option to go through the positive mood induction.

Mood grid Participants' arousal and valence were assessed by means of a two-dimensional ( 9 cells $\times 9$ cells) Likert scale (Russell et al., 1989). Participants were instructed to mark 1

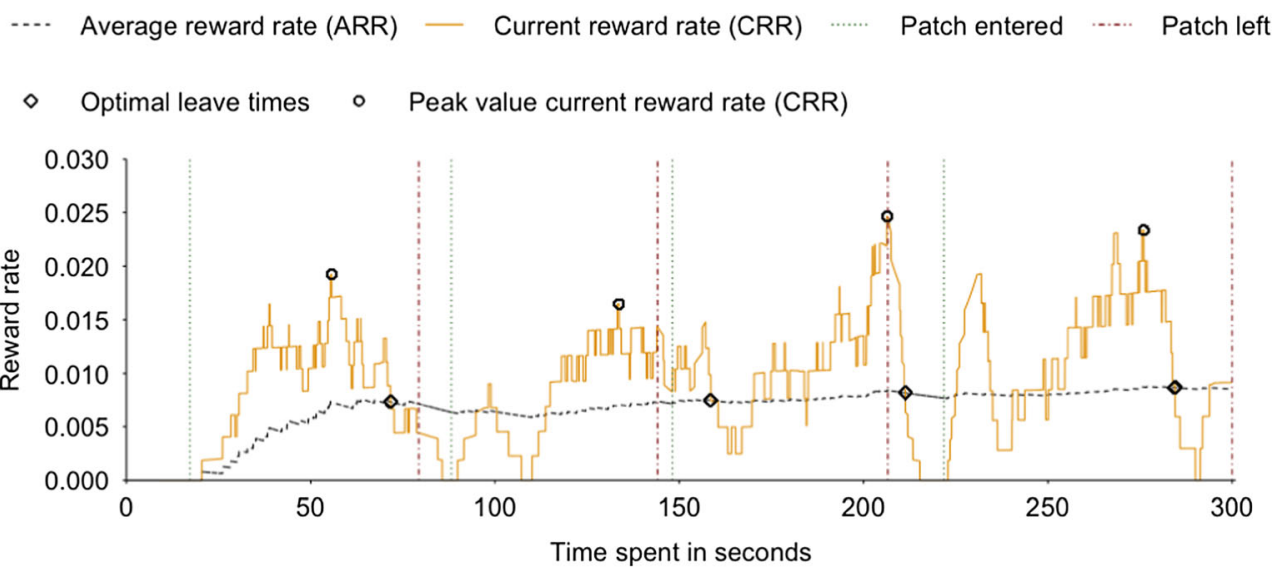

Fig. 2 ARR/CRR fitting procedure for one specific participant. To approximate optimal foraging behavior (following MVT), we calculated, separately for each participant and each time point, the current reward rate (CRR) and average reward rate (ARR) of the environment. Instances where the CRR and ARR intersect after a peak in the
CRR signal is detected (displayed as circles) reflect optimal leave times (OLTs; displayed as diamonds). These time points were compared with participants' actual leave times (i.e., ALTs; vertical red lines), which defined the leave time difference (LTD) parameter 
of 81 unique cells in the matrix. From left to right and from bottom to top, arousal and valence were scored from very sleepy $(-4)$ to highly aroused (+4) and from very unpleasant $(-4)$ to very pleasant $(+4)$, respectively. For example, if participants placed a cross in the upper-right corner of the grid, this would indicate feelings of excitement (high valence, high arousal). This relatively simple instrument has been found to have adequate reliability and validity (Russell et al., 1989). Participants were asked to fill out the mood grid a total of 6 times: before and after each of the foraging task runs (i.e., time points 1, 2, 5, and 6), and before and after the mood induction procedure (i.e., time points 3 and 4). The direct effect of the mood induction on mood was assessed by the arousal and valence ratings on the third and fourth mood grid.

\section{Statistical analyses}

All analyses were performed in the analysis software $\mathrm{R}(\mathrm{R}$ Core Team, 2019; Version 3.6.0), with the critical alpha value for significance being set to $p=0.05$, while correcting for multiple comparisons. Bayesian analyses with an uninformative model prior were additionally conducted to assess the strength of observed results. These analyses were performed using the BayesFactor package (Morey \& Rouder, 2018).

To assess whether mood ratings were significantly modulated by our mood induction manipulation, we subjected participants' arousal and valence ratings to two separate repeatedmeasures ANOVAs (ezANOVA from the R package $e z$ ) with Condition (excited vs. sad) as a between-subject factor and Time point ( 1 vs. 2 vs. 3 vs. 4 vs. 5 vs. 6) as a withinsubject factor. In case of a violation of the sphericity assumption, Greenhouse-Geisser corrected $d f$ and $p$ values are reported and error-terms are provided. For those analyses for which we had specific expectations regarding the directions of effects, one-tailed $t$-tests were performed. Associated $d f$ values were corrected by means of Welch's approximation in case of a violation of the assumption of homogeneity of variances, and $p$ values were accordingly corrected for multiple comparisons (Holm, 1979). To check whether groups did not differ in mood before the mood induction manipulation (time points 1 through 3), each of the RM-ANOVAs was followed up with planned post-hoc t-tests.

To assess whether (changes in) arousal and valence modulated behavior in the foraging task, we conducted three hierarchical multiple linear regression analyses. For the first analysis, we calculated the mean ratings provided on the mood grids presented at time points 1 and 2 (i.e., pre-mood induction ratings) and used these continuous variables to predict LTD scores for the first foraging run, while the mean ratings at time points 5 and 6 (i.e., post-mood induction ratings) were extracted as a measure of these parameters for the analysis on the LTD data of the foraging run post-mood induction. In order to test whether changes in arousal and valence were related to adjustments in foraging behavior, we fitted the third multiple linear regression model using difference scores for each of the abovementioned parameters; delta arousal (post-mood induction arousal minus pre-mood induction arousal), delta valence (post-mood induction valence minus pre-mood induction valence), and delta LTD (post-mood induction LTD minus premood induction LTD).

For each of the regression analyses, as a first step, a simple regression model was fitted. In a second step, these models were updated with the predictors' higher-order interaction term. These hierarchical models were compared using the anova function from the $\mathrm{R}$ package car. In case a more complex model did not outperform a more parsimonious model, we selected the model with the lowest BIC score. All models reported below adhered to the assumptions of independent errors (durbinWatsonTest from the R package car), multicollinearity of residuals (vif from $\mathrm{R}$ package car), normality of residuals, linearity of residuals, and homoscedasticity (Tabachnick \& Fidell, 2012).

Finally, for each of the foraging runs, we ran multiple hierarchical linear regression analyses to identify whether general foraging metrics were modulated by Condition (excited vs. sad) and arousal and valence scores. In particular, we focused on (1) the ratio of unique to total amount of patches visited, (2) the median time spent in patches, (3) the variability in patch leave times, (4) the mean travel time between patches, (5) the time elapsed before the first patch was entered, (6) the total area of the environment that was explored, and (7) the total amount of berries collected. Because these analyses are not within the main scope of the paper, these results can be consulted within the Supplementary Material.

\section{Results}

\section{Arousal ratings}

The RM-ANOVA on the arousal data with factors Condition (excited vs. sad) and Time point ( 1 vs. 2 vs. 3 vs. 4 vs. 5 vs. 6 ) showed a main effect of Condition $(F(1,115)=26.51, p<$ $\left.0.001, \eta \mathrm{p}^{2}=0.19\right)$ and a main effect of Time point $(F(3.57$, $575)=8.42, p<0.001, \varepsilon=0.71, \eta p^{2}=0.07$ ) (Supplementary Table 2). Overall, arousal ratings were higher for participants in the excited condition $(M=1.47, S D=1.40)$ compared with participants in the sad condition $(M=0.52, S D=1.46$, see Supplementary Figures 2a-f for the associated 2D-heatmaps). Moreover, and as expected, a significant Condition $\times$ Time point interaction was observed $(F(3.57,575)=24.07, p<$ 0.001, $\varepsilon=0.71, \eta p^{2}=0.17$, Fig. 3). Along similar lines, the Bayesian RM-ANOVA showed strong evidence in favor of the alternative hypothesis $\left(\mathrm{BF}_{10}=1.51 \times 10^{27}\right)$. More precisely, the estimated Bayes factor suggests that the data are $1.51 \times 10^{27}$ times more likely under the alternative hypothesis - namely 
$\longrightarrow$ Excited $-\rightarrow--$ Sad
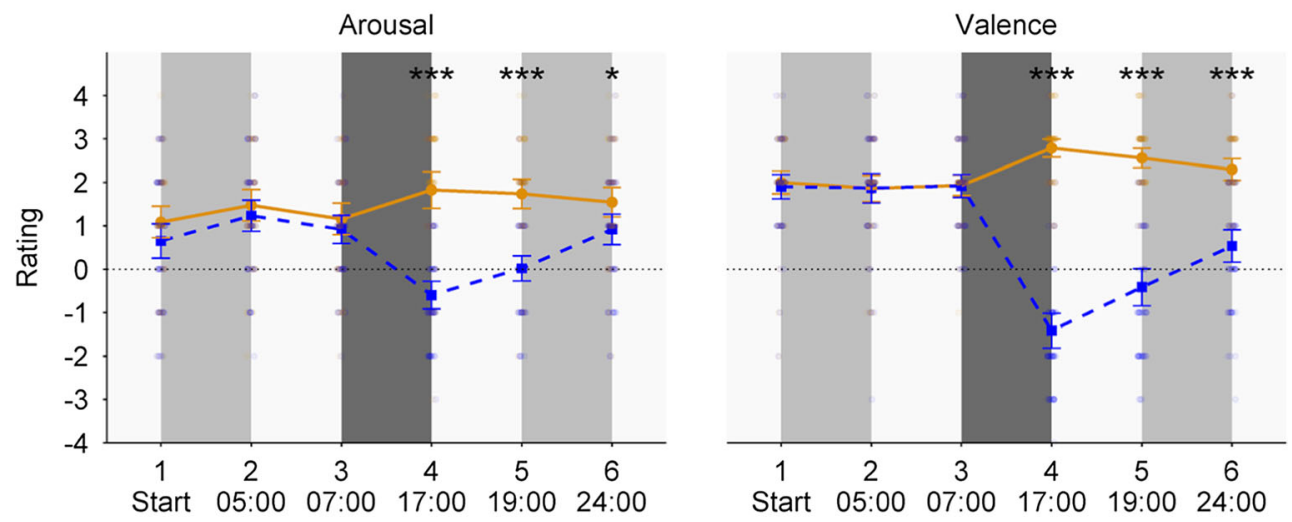

Time point and average time spent in minutes since first mood/arousal grid

Fig. 3 Self-reported arousal and valence ratings before and after each of the foraging runs (time points 1, 2, 5 and 6), and before and after the mood induction procedure (time points 3 and 4). The light-grey boxes indicate the moments at which participants performed the pre-mood induction (i.e., first light-grey box) and post-mood induction foraging runs (i.e., second light-grey box). The dark-grey box indicates the moment where

that the factors Condition and Time point affect arousal ratings - compared with the null hypothesis.

Post-hoc $t$-tests (Holm-corrected) revealed that arousal ratings for the excited and sad conditions did not significantly differ preceding the mood induction manipulation (time points 1 through 3, $|t| \mathrm{s}<1.62, p \mathrm{~s}_{\text {corr }}>0.16, d \mathrm{~s}<0.30,0.3<\mathrm{BFs}_{01}<$ 3 ). In contrast, for time points 4,5 , and 6 , arousal ratings were significantly larger in the excited condition compared with the sad condition $\left(|t| \mathrm{s}>2.57, p \mathrm{~s}_{\text {corr }}<0.024, d \mathrm{~s}>0.47, \mathrm{BFs}_{10}>\right.$ 7.25; Supplementary Table 4).

Finally, one-tailed paired-sample $t$-tests on the ratings provided at time points 3 and 4 confirmed that arousal ratings significantly increased for participants in the excited condition $(|t|(56)=2.85, p=0.003, d=0.45,95 \%$ confidence interval [CI] [0.07, 0.82], $\left.\mathrm{BF}_{10}=5.48\right)$, whereas these ratings significantly decreased for participants in the sad condition $(|t|(59)=$ $8.01, p<0.001, d=-1.22,95 \%$ CI $[-0.82,-1.61], \mathrm{BF}_{10}=$ $\left.1.83 \times 10^{8}\right)$.

\section{Valence ratings}

The RM-ANOVA on the valence data with factors Condition (excited vs. sad) and Time point (1 vs. 2 vs. 3 vs. 4 vs. 5 vs. 6 ) showed a main effect of Condition $(F(1,115)=94.20, p<$ $\left.0.001, \eta \mathrm{p}^{2}=0.45\right)$ and a main effect of Time point $(F(3.73$, $\left.575)=39.25, p<0.001, \varepsilon=0.75, \eta p^{2}=0.25\right)$ (Supplementary Table 3). Overall, valence ratings were higher for participants in the excited condition $(M=2.24, S D=0.99)$ compared with participants in the sad condition $(M=0.73, S D=1.87$, Supplementary Figures $2 \mathrm{a}-\mathrm{f}$ for the associated 2D-heatmaps). Moreover, the analysis showed a significant Condition $\times$ Time point interaction $(F(3.73,575)=115.22, p<0.001, \varepsilon=0.75$, the mood induction procedure was presented. Each of these time points has additionally been labeled as the average amount of elapsed minutes since the presentation of the first mood grid (i.e., time point 1). Individual data points are reported. Error bars represent $95 \%$ between-subject CIs. $* p<0.05, * * * p<0.001$, Holm-corrected for multiple comparisons

$\eta p^{2}=0.50$; Fig. 3). Along similar lines, the Bayesian RMANOVA showed strong evidence in favor of the alternative hypothesis $\left(\mathrm{BF}_{10}=8.89 \times 10^{110}\right)$. The estimated Bayes factor suggests that the data are $8.89 \times 10^{110}$ times more likely under the alternative hypothesis - namely that the factors Condition and Time point affect valence ratings - compared with the null hypothesis.

Post-hoc $t$-tests (Holm-corrected) revealed that valence ratings for the excited and sad conditions did not significantly differ preceding the mood induction manipulation (time points 1 through $\left.3,|t| \mathrm{s}<0.52, p \mathrm{~s}_{\text {corr }}>0.91, d \mathrm{~s}<0.10, \mathrm{BFs}_{01}>3.28\right)$. In contrast, for time points 4,5 , and 6 , valence ratings were significantly larger in the excited condition compared with the sad condition $\left(|t| \mathrm{s}>7.86, p \mathrm{~s}_{\text {corr }}<0.001, d \mathrm{~s}>1.44, \mathrm{BFs}_{10}>\right.$ $3.82 \times 10^{9}$; Supplementary Table 4).

Finally, one-tailed, paired-sample $t$-tests on the ratings provided at time points 3 and 4 confirmed that valence ratings significantly increased for participants in the excited condition $\left(|t|(56)=7.42, p<0.001, d=1.04,95 \%\right.$ CI $[0.64,1.43], \mathrm{BF}_{10}$ $=1.54 \times 10^{7}$ ), whereas these ratings significantly decreased for participants in the sad condition $(|t|(59)=15.42, p<0.001, d=$ $-2.53,95 \%$ CI $\left.[-2.04,-3.01], \mathrm{BF}_{10}=1.88 \times 10^{19}\right)$.

\section{Validity of condition classifications}

Interestingly, as can be distilled from Fig. 3, arousal and valence levels were fairly high for participants in the sad mood condition, both before and after mood induction. Specifically, although ratings of most participants in the excited mood condition fell into the expected upper half of the scores, indicating high arousal and valence (50/57 participants), a dissimilar pattern was observed for participants in the sad mood 
condition, as most participants still scored in the upper half of the grid, indicating high arousal and valence, and only a small part (14/60 participants) scored relatively low on these measures. Put differently, although our manipulation was successful in inducing variability in mood between groups, participants' arousal and valence ratings did not necessarily conform to the pattern of ratings as expected from the predefined classifications (Van Steenbergen et al., 2010). Because we were interested in interindividual adjustments of control states as induced by our mood manipulation, we treated the selfreported arousal and valence ratings as continuous predictors within all subsequent analyses, rather than comparing discrete groups based on mood induction conditions. However, the interested reader can consult the Supplementary Material for all analyses incorporating the factor Condition. It is worth mentioning that, irrespective of whether the factor Condition was added to the models, the below described effects of interest remain unaltered.

\section{Foraging task metrics as predicted by mood}

Three hierarchical multiple linear regression analyses were conducted to reveal whether (changes in) arousal and/or valence modulated participants' LTD scores. Before model specification, four participants were removed, as their LTD value on either the pre-mood induction or post-mood induction foraging run exceeded the interquartile range by factor 2.2. Even though the below reported results did not change when including these LTD outliers, we nevertheless decided to exclude these observations as such omissions eliminated violations of model assumptions. Importantly, for each of the analyses reported below, ANOVAs revealed that updating the models did not significantly increase the model fits (all $F_{\mathrm{s}}(1,109)<3.88$, all $\left.p \mathrm{~s}>0.051\right)$. All below-reported $p$ values have been multiplied by a factor 3 to correct statistically for the total amount of performed regression analyses.

The regression analysis on the relationship between LTD and arousal and valence ratings pre-mood induction did not reveal any significant association $\left(F(2,110)=3.05, p_{\text {corr }}=\right.$ $0.15, R^{2}=0.04 ; \beta=-0.14,95 \%$ CI $[-0.33,0.04],|t|(110)=$ $1.51, p_{\text {corr }}=0.39 ; \beta=-0.16,95 \% \mathrm{CI}[-0.34,0.03],|t|(110)=$ $1.67, p_{\text {corr }}=0.30$, for arousal and valence ratings, respectively). The Bayesian linear regression analysis did not reveal sufficient evidence $(0.3<\mathrm{BF}<3)$ in favor of the null nor the alternative hypothesis $\left(\mathrm{BF}_{10}=0.80\right)$.

Similarly, the second regression analysis did not reveal any relationship between the mood predictors and LTD post-mood induction $\left(F(2,110)=0.41, p_{\text {corr }}=1.0, R^{2}=-0.01 ; \beta=0.00\right.$, $95 \%$ CI $[-0.21,0.21],|t|(110)=0.01, p_{\text {corr }}=1.0$ and $\beta=$ $-0.09,95 \%$ CI $[-0.30,0.12],|t|(110)=0.82, p_{\text {corr }}=1.0$ for arousal and valence ratings, respectively). The Bayesian linear regression analysis showed strong evidence $\left(\mathrm{BF}_{01}=11.79\right)$ in favor of the null hypothesis. Specifically, the estimated Bayes factor suggested that the data were 11.79 times more likely under the hypothesis that post-mood induction LTD and arousal and valence ratings post-mood induction were not related.

Finally, and interestingly enough, the third regression analysis revealed that delta LTD scores were significantly predicted by both delta arousal and delta valence ratings $(F(2,110)=$ $6.79, p_{\text {corr }}=0.005, R^{2}=0.09$ ). Specifically, while an increase in arousal was associated with an increase in exploratory foraging behavior $(\beta=0.24,95 \%$ CI $[0.05,0.44],|t|(110)=2.51$, $\left.p_{\text {corr }}=0.041\right)$, an increase in valence was associated with an increase in exploitative behavior $(\beta=-0.33,95 \% \mathrm{CI}[-0.53$, $-0.14],|t|(110)=3.45, p_{\text {corr }}=0.002$; Fig. 4). Importantly, the Bayesian linear regression analysis showed strong evidence in favor of the alternative hypothesis $\left(\mathrm{BF}_{10}=17.65\right)$. More precisely, the estimated Bayes factor suggested that the data were 17.65 times more likely under the alternative hypothesisnamely the hypothesis that changes in arousal and/or valence significantly correlated with adjustments in foraging behavior - compared with the null hypothesis.

\section{Discussion}

Our main research goal was to study whether and how the regulation of exploration and exploitation relates to the regulation of the persistence-flexibility trade-off. To fill this gap, we induced, between-participants, two types of mood that are assumed to differ in arousal and valence (i.e., excited vs. sad), and subsequently tested whether these mood states differentially affected individual tendencies towards exploration/ exploration as measured by means of a foraging task. As previously alluded to, our research aim can be divided into three questions, which we will address in turn.

The answer to the first question, whether changes in arousal and/or valence systematically affect exploration and exploitation, is clearly yes: not only did we manage to successfully induce the expected mood changes in our participants, but we also were able to show that these changes had an effect on participants' tendency to explore/exploit in the foraging task. Specifically, adjustments in both arousal and valence were associated with changes in foraging behavior: while increases in arousal significantly correlated with increased levels of exploration, increases in valence substantially increased exploitative foraging behavior. From a computational perspective, the induction of a positive or negative mood could modulate either information integration or the decision mechanism. There is evidence from other domains to suggest that primacy and recency effects are affected by mood (see Forgas, 2011 for a social psychology approach), which may cause the difference in foraging behavior. However, such a conclusion seems problematic, as it can be argued that exploratory behavior from this point of view could be induced by an overestimation 

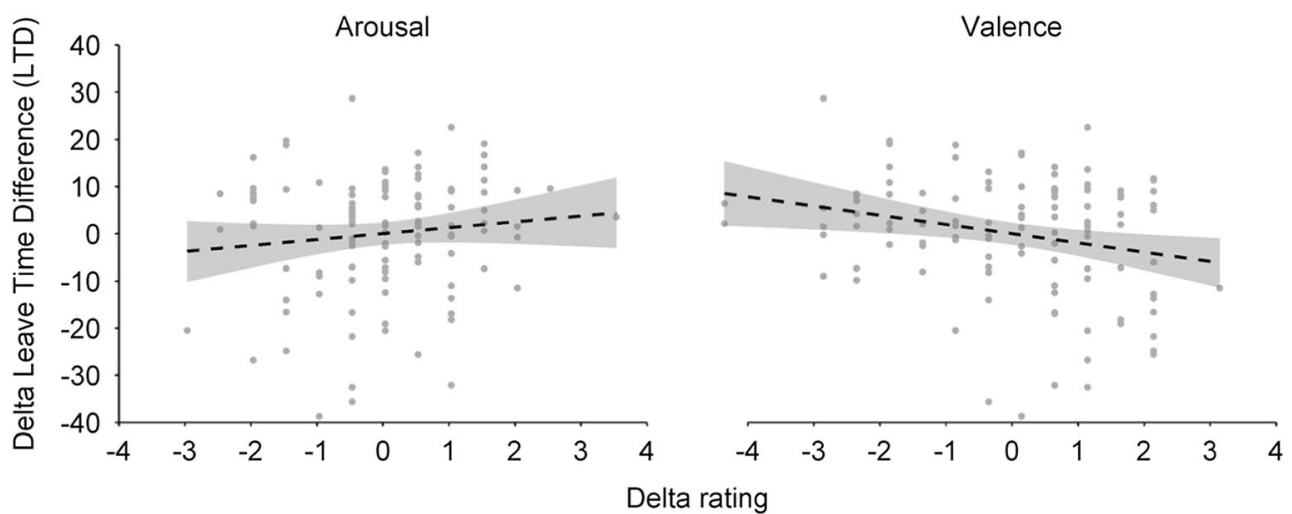

Fig. 4 Delta arousal and valence ratings (post-mood induction ratings minus pre-mood induction ratings) predict delta leave time differences scores (post-mood induction LTD minus pre-mood induction LTD). Specifically, while increases in arousal are associated with increases in exploratory behavior, increases in valence are correlated with increases in exploitative behavior. Individual data points are reported. The bandwidths represent $95 \%$ between-subject CIs

on flexibility and persistence, or both. Even though we did not directly assess physiological arousal in the current study, our findings suggest that both arousal and valence differently and uniquely contribute to the tendency to explore or exploit. If we speculate arousal is indicative of the noradrenergic system (Aston-Jones \& Cohen, 2005), whereas valence may reflect changes in the dopaminergic system (Goschke \& Bolte, 2014), our findings would be consistent with Cohen et al. (2007), who speculated that both systems might be involved in regulating control biases. This proposition fits well with increasing evidence from neurochemical and physiological studies suggesting interesting interactions between NE and DA and their sources (i.e., locus coeruleus and the ventral tegmental area) in regulating various kinds of processes (Ranjbar-Slamloo \& Fazlali, 2020). However, as the functional role of these interactions still remains to be better understood, and the current study does not provide data to substantiate direct inferences on the involvement of neuromodulators, future work should focus on filling these gaps by including direct manipulations of catecholaminergic activity (Buffalari \& Grace, 2007; Frank et al., 2009; Frank \& Fossella, 2011; Kayser et al., 2015). This is particularly advised when considering the results presented here: a modulation of foraging behavior was only observed when accounting for participants' performance pre-mood induction, an observation that emphasizes the importance of studying inter- and intra-individual differences in control processes (Mekern et al., 2019).

When interpreting our results, one important caveat has to be addressed. Specifically, although our experimental paradigm enabled us to examine the potential role of both arousal and valence in modulating the exploration-exploitation balance, mood induction protocols traditionally incorporate four conditions, reflecting excited (high arousal, high valence), sad (low arousal, low valence), anxious (high arousal, low valence), and calm (low arousal, high valence) induction conditions (Van Steenbergen et al., 2010). Because our main 
rationale to use the mood induction paradigm was to maximize the variance of mood impact on the explorationexploitation trade-off, our protocol merely incorporated those two (out of four) unique dimensions that most likely resulted in the largest variability in arousal and valence scores. This might have resulted in an underestimation of the distinct contributions of, and interactions between, arousal and valence. However, even though the observed effects call for a more systematic investigation of the contributions of both $\mathrm{NE}$ and DA in regulating both the exploration-exploitation and persistence-flexibility balance, this does not render the current results any less interesting.

Concluding, our study shows that mood plays some role in the level of exploration-exploitation during foraging behavior, but that the directions of effects do not directly map onto the mood effects observed for modulation of flexible-persistent control tendencies. Together, this provides first insights into how processes related to arousal and valence differentially modulate foraging behavior. Considering links between mood and catecholaminergic neural mechanisms, our results might suggest a role of both noradrenaline and dopamine in regulating the exploration/exploitation trade-off, and signify that the relationship between exploration-exploitation and flexibilitypersistence might be more complicated than previously assumed.

Acknowledgments The authors thank André van Arkel, Guusje Dijkman, Alice Hill, and Annoek van Koten for their help in data collection. Moreover, they thank Vera Mekern for her help in beautifully illustrating the foraging paradigm. Finally, thanks to Thomas Hills and Roberta Sellaro for their valuable insights regarding the design of the foraging paradigm. This work was supported by the European Research Council (ERC Advanced Grant: grant agreement No 694722 [Metacontrol] to B. Hommel) under the European Union's Horizon 2020 research and innovation program.

Author contributions Author contributions are coded according to the CRediT taxonomy: https://casrai.org/credit/ (Allen et al., 2014).

Conceptualization: $\mathrm{BH}, \mathrm{ZS}$

Data curation: RvD

Formal analysis: RvD, RdK

Funding acquisition: $\mathrm{BH}$

Investigation: $\mathrm{RvD}, \mathrm{ZS}$

Methodology: RvD, RdK, ZS

Project administration: RvD, ZS

Resources: RvD, BH, ZS

Software: RvD, RdK

Supervision: ZS

Validation: RvD

Visualization: RvD

Writing - editorial role: RvD, ZS

Writing - original draft: RvD, BH

Writing - review \& editing: RvD, RdK, BH, ZS

\section{Declarations}

Conflicts of interest None
Open Access This article is licensed under a Creative Commons Attribution 4.0 International License, which permits use, sharing, adaptation, distribution and reproduction in any medium or format, as long as you give appropriate credit to the original author(s) and the source, provide a link to the Creative Commons licence, and indicate if changes were made. The images or other third party material in this article are included in the article's Creative Commons licence, unless indicated otherwise in a credit line to the material. If material is not included in the article's Creative Commons licence and your intended use is not permitted by statutory regulation or exceeds the permitted use, you will need to obtain permission directly from the copyright holder. To view a copy of this licence, visit http://creativecommons.org/licenses/by/4.0/.

\section{References}

Akbari Chermahini, S., \& Hommel, B. (2012). Creative mood swings: divergent and convergent thinking affect mood in opposite ways. Psychological Research, 76(5), 634-640. https://doi.org/10.1007/ s00426-011-0358-z

Allen, L., Scott, J., Brand, A., Hlava, M., \& Altman, M. (2014). Publishing: Credit where credit is due. Nature News, 508(7496), 312.

Aston-Jones, G., \& Cohen, J. D. (2005). An integrative theory of locus coeruleus-norepinephrine function: adaptive gain and optimal performance. Annual Review of Neuroscience, 28(1), 403-450. https:// doi.org/10.1146/annurev.neuro.28.061604.135709

Atkinson, J. W., \& Birch, D. (1970). The dynamics of action. John Wiley.

Buffalari, D. M., \& Grace, A. A. (2007). Noradrenergic modulation of basolateral amygdala neuronal activity: Opposing influences of $\alpha-2$ and $\beta$ receptor activation. Journal of Neuroscience, 27(45), 1235812366. https://doi.org/10.1523/JNEUROSCI.2007-07.2007

Cahilla, L., \& Alkireb, M. T. (2003). Epinephrine enhancement of human memory consolidation: Interaction with arousal at encoding. Neurobiology of Learning and Memory, 79, 194-198.

Charnov, E. L. (1976). Optimal foraging, the marginal value theorem. Theoretical Population Biology, 9(2), 129-136. https://doi.org/10. 1016/0040-5809(76)90040-X

Cohen, J. D., McClure, S. M., \& Yu, A. J. (2007). Should I stay or should I go? How the human brain manages the trade-off between exploitation and exploration. Philosophical Transactions of the Royal Society B: Biological Sciences, 362(1481), 933-942. https://doi. org/10.1098/rstb.2007.2098

Cools, R., \& D'Esposito, M. (2011). Inverted-U-shaped dopamine actions on human working memory and cognitive control. Biological Psychiatry, 69(12), e113-e125. https://doi.org/10.1016/j.biopsych. 2011.03.028

Dreisbach, G., \& Goschke, T. (2004). How positive affect modulates cognitive control: reduced perseveration at the cost of increased distractibility. Journal of Experimental Psychology: Learning Memory and Cognition, 30(2), 343-353. https://doi.org/10.1037/ 0278-7393.30.2.343

Dreisbach, G., Müller, J., Goschke, T., Strobel, A., Schulze, K., Lesch, K.-P., \& Brocke, B. (2005). Dopamine and cognitive control: the influence of spontaneous eyeblink rate and dopamine gene polymorphisms on perseveration and distractibility. Behavioral Neuroscience, 119(2), 483-490. https://doi.org/10.1037/07357044.119.2.483

Durstewitz, D., \& Seamans, J. K. (2008). The dual-state theory of prefrontal cortex dopamine function with relevance to Catechol-OMethyltransferase genotypes and schizophrenia. Biological Psychiatry, 64(9), 739-749. https://doi.org/10.1016/j.biopsych. 2008.05.015 
Eich, E., Ng, J. T., Macaulay, D. P. A. D., Percy, A. D., \& Grebneva, I. (2007). Combining music with thought to change mood. Handbook of emotion elicitation and assessment, 124-136.

Erdfelder, E., Faul, F., \& Buchner, A. (1996). GPOWER: A general power analysis program. Behavior research methods, instruments, \& computers, 28(1), 1-11.

Forgas, J. P. (2011). Can negative affect eliminate the power of first impressions? Affective influences on primacy and recency effects in impression formation. Journal of Experimental Social Psychology, 47, 425-429.

Frank, M. J., Doll, B. B., Oas-Terpstra, J., \& Moreno, F. (2009). Prefrontal and striatal dopaminergic genes predict individual differences in exploration and exploitation. Nature Neuroscience, 12(8), 1062-1068. https://doi.org/10.1038/nn.2342

Frank, M. J., \& Fossella, J. A. (2011). Neurogenetics and pharmacology of learning, motivation, and cognition. Neuropsychopharmacology, 36(1), 133-152. https://doi.org/10.1038/npp.2010.96

Fröber, K., \& Dreisbach, G. (2014). The differential influences of positive affect, random reward, and performance-contingent reward on cognitive control. Cognitive, Affective and Behavioral Neuroscience, 14(2), 530-547. https://doi.org/10.3758/s13415-014-0259-x

Goschke, T. (2003). Voluntary action and cognitive control from a cognitive neuroscience perspective. In S. Maasen, W. Prinz, \& G. Roth (Eds.), Voluntary action. An issue at the interface of nature and culture (pp. 49-85). Oxford University Press.

Goschke, T., \& Bolte, A. (2014). Emotional modulation of control dilemmas: The role of positive affect, reward, and dopamine in cognitive stability and flexibility. Neuropsychologia, 62, 403-423. https://doi.org/10.1016/j.neuropsychologia.2014.07.015

Hills, T. T. (2006). Animal foraging and the evolution of goal-directed cognition. Cognitive Science, 30(1), 3-41. https://doi.org/10.1207/ s15516709 $\operatorname{cog} 0000 \quad 50$

Hills, T. T., \& Dukas, R. (2012). The Evolution of cognitive search. Cognitive Search: Evolution, Algorithms, and the Brain, 11-24. https://doi.org/10.1111/j.1756-8765.2009.01078.x

Hills, T. T., Todd, P. M., \& Goldstone, R. L. (2008). Search in external and internal spaces: Evidence for generalized cognitive search processes. Psychological Science, 19(8), 802-808. https://doi.org/10. 1111/j.1467-9280.2008.02160.x

Hills, T. T., Todd, P. M., \& Goldstone, R. L. (2010). The central executive as a search process: priming exploration and exploitation across domains. Journal of Experimental Psychology, 139(4), 590-609. https://doi.org/10.1037/a0020666.

Holm, S. (1979). A simple sequentially rejective multiple test procedure. Scandinavian Journal of Statistics, 6(2), 65-70.

Hommel, B. (2015). Between persistence and flexibility: the Yin and Yang of action Control. Advances in Motivation Science, 2, 33 67. https://doi.org/10.1016/bs.adms.2015.04.003

Hommel, B. (2019). Affect and control: a conceptual clarification. International Journal of Psychophysiology, 144, 1-6.

Hommel, B., \& Colzato, L. S. (2017). The social transmission of metacontrol policies: Mechanisms underlying the interpersonal transfer of persistence and flexibility. Neuroscience and Biobehavioral Reviews, 81, 43-58. https://doi.org/10.1016/j. neubiorev.2017.01.009

Jefferies, L. N., Smilek, D., Eich, E., \& Enns, J. T. (2008). Emotional valence and arousal interact in attentional control. Psychological Science, 19(3), 290-295. https://doi.org/10.1111/j.1467-9280. 2008.02082.x

Jepma, M., \& Nieuwenhuis, S. (2011). Pupil diameter predicts changes in the exploration-exploitation trade-off: evidence for the adaptive gain theory. Journal of Cognitive Neuroscience, 23(7), 1587-1596. https://doi.org/10.1162/jocn.2010.21548

Kayser, A. S., Mitchell, J. M., Weinstein, D., \& Frank, M. J. (2015). Dopamine, locus of control, and the exploration-exploitation tradeoff. Neuropsychopharmacology, 40(2), 454-462. https://doi. org/10.1038/npp.2014.193

Lenow, J. K., Constantino, S. M., Daw, N. D., \& Phelps, E. A. (2017). Chronic and acute stress promote overexploitation in serial decision making. Journal of Neuroscience, 37(23), 5681-5689. https://doi. org/10.1523/JNEUROSCI.3618-16.2017

Marković, D., Goschke, T., \& Kiebel, S.J. (2019). Meta-control of the exploration-exploitation dilemma emerges from probabilistic inference over a hierarchy of time scales. bioRxiv, 847566. https://doi. org $/ 10.1101 / 847566$

Mather, M., Clewett, D., Sakaki, M., \& Harley, C. W. (2016). Norepinephrine ignites local hot spots of neuronal excitation: How arousal amplifies selectivity in perception and memory. Behavioral and Brain Sciences, 39. https://doi.org/10.1016/j.physbeh.2017.03. 040

Mekern, V. N., Sjoerds, Z., \& Hommel, B. (2019). How metacontrol biases and adaptivity impact performance in cognitive search tasks. Cognition, 182, 251-259. https://doi.org/10.1016/j.cognition.2018. 10.001

Morey, R. D., \& Rouder, J. N. (2018). BayesFactor: Computation of Bayes factors for common designs. R package version 0.9.12-4.2. URL https://CRAN.R-project.org/package=BayesFactor

R Core Team. (2019). R: A language and environment for statistical computing. R Foundation for Statistical Computing, Vienna, Austria. URL https://www.R-project.org/.

Ranjbar-Slamloo, Y., \& Fazlali, Z. (2020). Dopamine and noradrenaline in the brain; overlapping or dissociate functions? Frontiers in Molecular Neuroscience, 12, 1-8. https://doi.org/10.3389/fnmol. 2019.00334

Riefer, P. S., Prior, R., Blair, N., Pavey, G., \& Love, B. C. (2017). Coherency-maximizing exploration in the supermarket. Nature $h u$ man behaviour, 1(1), 1-4. https://doi.org/10.1038/s41562-016-0017

Russell, J. A., Weiss, A., \& Mendelsohn, G. A. (1989). Affect grid: a single-item scale of pleasure and arousal. Journal of Personality and Social Psychology, 57(3), 493-502. https://doi.org/10.1037/00223514.57.3.493

Sheehan, D. V., Lecrubier, Y., Sheehan, K. H., Amorim, P., Janavs, J., Weiller, E., ... \& Dunbar, G. C. (1998). The Mini-International Neuropsychiatric Interview (MINI): the development and validation of a structured diagnostic psychiatric interview for DSM-IV and ICD-10. The Journal of Clinical Psychiatry, 59(20), 22-33.

Tabachnick, B. G., \& Fidell, L. S. (2012). Chapter 13 principal components and factor analysis. Using multivariate statistics. London: Pearson.

Terbeck, S., Savulescu, J., Chesterman, L. P., \& Cowen, P. J. (2016). Noradrenaline effects on social behaviour, intergroup relations, and moral decisions. Neuroscience and Biobehavioral Reviews, 66, 54 60. https://doi.org/10.1016/j.neubiorev.2016.03.031

Todd, P.M., \& Hills, T.T. (2020). Foraging in mind. Current Directions in Psychological Science, 29(3), 309-315. https://doi.org/10.1177/ 0963721420915861

Van Steenbergen, H., Band, G. P. H., \& Hommel, B. (2010). In the mood for adaptation: How affect regulates conflict-driven control. Psychological Science, 21(11), 1629-1634. https://doi.org/10. $1177 / 0956797610385951$

Vinckier, F., Rigoux, L., Oudiette, D., \& Pessiglione, M. (2018). Neurocomputational account of how mood fluctuations arise and affect decision making. Nature Communications, 9(1), 1-12. https://doi. org/10.1038/s41467-018-03774-z

Wolfe, J. M. (2013). When is it time to move to the next raspberry bush? Foraging rules in human visual search. Journal of Vision, 13(3), 117. https://doi.org/10.1167/13.3.10

Publisher's note Springer Nature remains neutral with regard to jurisdictional claims in published maps and institutional affiliations. 